\title{
DNA Microarrays in Medicine: Can the Promises Be Kept?
}

\author{
Tarik Möröy* \\ Institut für Zellbiologie (Tumorforschung), IFZ, Universitätsklinikum Essen, \\ Virchowstrasse 173, D-45122 Essen, Germany
}

DNA microarrays can be used to measure the steady state mRNA level of most or all genes of a cell and deliver a quantification of the transcriptional profile on a genomwide scale. The promises that come along with this new technology are a rapid access to molecular pathways in biology, a more precise diagnosis and prognosis of diseases, a better understanding of drug action, the ability to better define therapeutic strategies, and many more. DNA microarrays may also build a new bridge on which biologists and clinicians can meet and bring to fruition what has been termed "translational medicine" for the benefit of patients.

DNA microarrays are built with snippets of DNA representing almost the entire genetic information of an organism on a few square centimeters. They are commercially available or can readily be made in laboratories with the right equipment. No matter whether oligonucleotides are synthesized on glass by a sophisticated technology first developed for the semiconductor industry or whether longer PCR products are simply dotted onto solid surfaces, the scientific community has a new and powerful tool to measure gene activity. To uncover correlations between biological phenomena or medical conditions and genetic regulatory mechanisms, researchers no longer have to rely on single gene analysis. However, the availability of DNA microarrays not only opens the door to new types of experiments but also delivers a new type of data. Comparing the expression level of thousands of genes at the same time cannot be carried out with the same skills required to correctly interpret autoradiograms. Computer power, the right software, and the advice of experts in the bioinformatic field will be necessary.

The possibilities and promises of DNA microarrays are more than numerous. Cancer research was the first prominent field that used the new technology. Over 20 years of basic research have laid the ground for a molecular concept of cancer as a "genetic disease." But it also demonstrated the immense complexity of each of the over 100 different diseases that can be classified under the general term "cancer." Now, expression profiling of tumors with DNA microarrays will help to better define a particular tumor type and distinguish it from others. The first experiments have already pointed the way: gene expression profiles of tumor samples have been correlated with clinical parameters. The goal is clear: with the help of cluster algorithms, every tumor should be labeled with a molecular signature drawn from numerous expression profiles.

By the same token, DNA microarrays will be used to assess the effects of drugs on the transcriptional activity of target cells or tissues. For basic researchers this should be of immense help to understand processes such as drug induced apoptosis, drug induced DNA damage and repair, or other more general aspect of gene regulation by small molecules. For the pharmaceutical industry, a correlation between gene activity and effects of candidate compounds may help to eliminate those with severe side effects before they enter clinical trials. Also, it will help to define the effects of those drugs already in clinical use. This application of DNA microarray technology falls under the roof of a new discipline for which the label "pharmacogenomics" has already been coined.

Other DNA microarrays will be, or already have been, engineered that allow to test for the presence of specific germs in tissues or body fluids. It will be possible to identify the bacteria or viruses responsible for infectious disease much faster and with higher precision than before. Also the traces that genetically modified organisms may leave in industrial food products may be testable on a large scale. Specifically designed DNA microarrays, that use "on-chip" PCR reactions for all known genes used for the genetic engineering of plant and animals in the food industry, are already on the drawing boards.

Human geneticists will use specific DNA microarrays to detect the mutations underlying monogenetic, hereditary diseases or to interrogate for more general predispositions of a number of conditions. In addition to gene expression arrays, it is already possible to measure the presence or absence of specific single nucleotide polymorphisms (SNP) in the human genome. Researches worldwide and also the Biotech industry have intensified their search for human SNPs. Soon we will be confronted with a database describing all the estimated 3 million human SNPs. It may not be too far fetched to speculate that SNP analysis by DNA microarray technology 
may allow in the future to predict onset, prognosis, and outcome of diseases that are not yet clinically manifest at the time of analysis. Moreover, the protagonists of SNP analysis predict the emergence of a new kind of individual, predictive medicine; even a revolution in medicine and cancer therapy is proclaimed. With SNP analysis and gene expression profiling, it should be possible to design individual therapies tailored for a single patient after his/her individual responses towards drugs, radiation, or other types of therapies.

DNA microarrays are a new and exciting technology. Similar to all new innovations this technology comes with many promises for patients, doctors, and researchers alike. Some of the promises will be kept, some will turn out to be illusions, and others may turn out to be very difficult to handle without additional legislation. Cancer classification has begun and the results have already helped to reevaluate therapy and prognosis in certain cases. Similarly, basic research has profited from the new technology. In this regard, some promises have been kept. We have to wait and see to what extent all the other applications of DNA microarray technology will live up to the their expectations. The new knowledge of whole organism sequences and the application of genomic technology as DNA microarrays have opened a new and exciting era of biology. Despite the large progress that has been made in understanding biological systems in the past decades, we are again at a new beginning.

Tarik Möröy

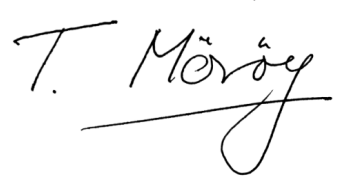

* E-mail: moeroey@uni-essen.de 

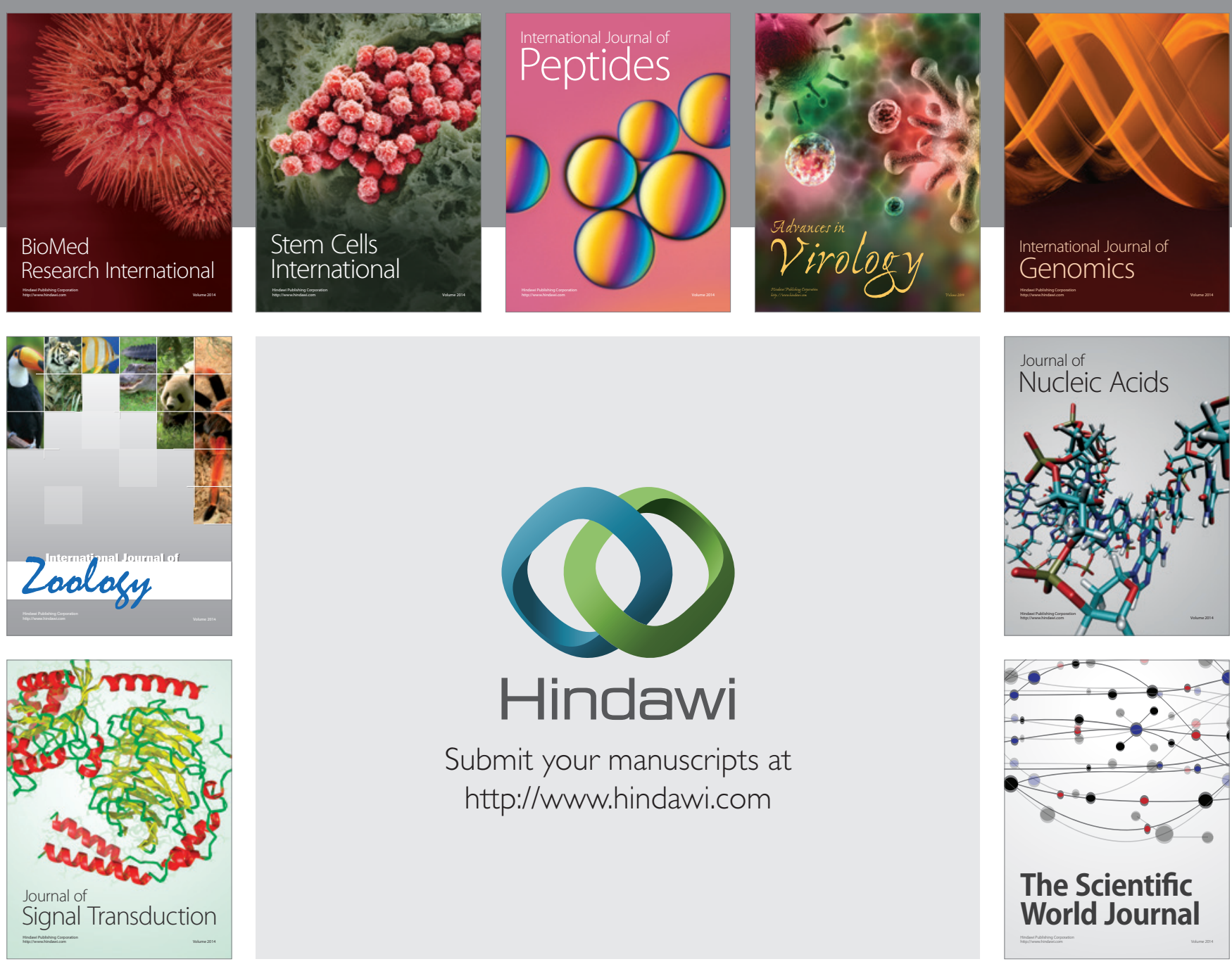

Submit your manuscripts at

http://www.hindawi.com
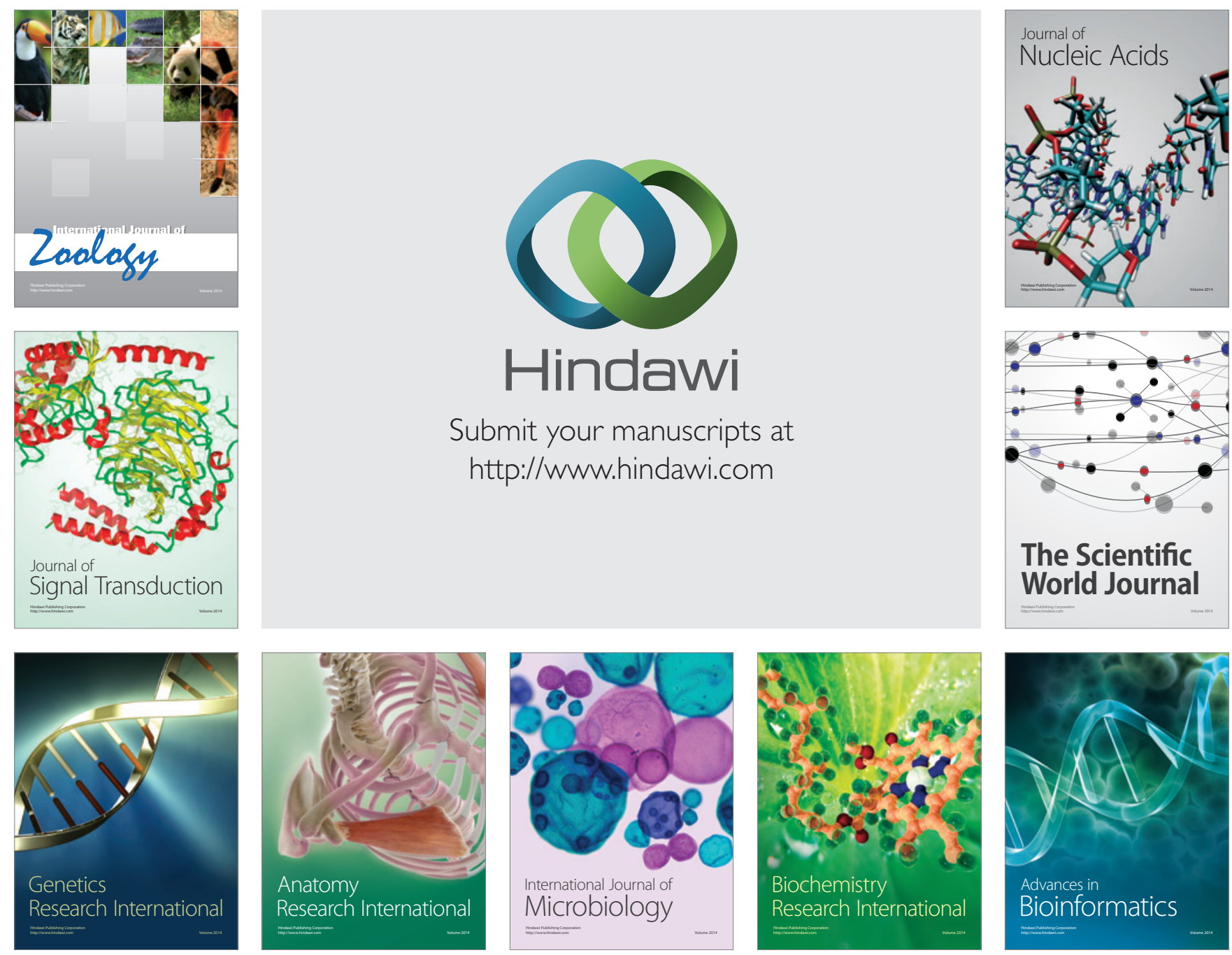

The Scientific World Journal
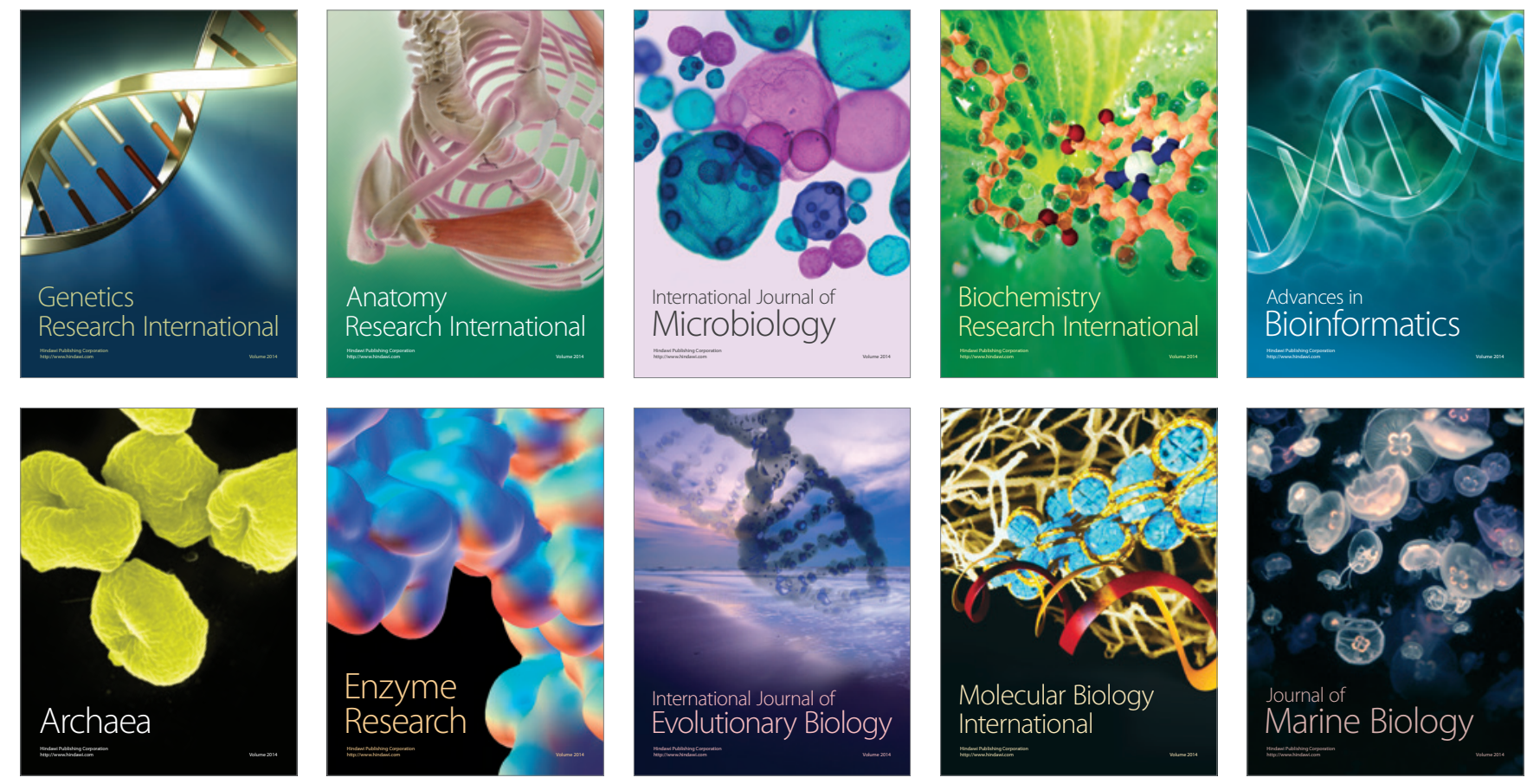